\title{
Spectral index maps of the radio halos in Abell 665 and Abell 2163
}

\author{
L. Feretti ${ }^{1}$, E. Orrù ${ }^{1}$, G. Brunetti ${ }^{1}$, G. Giovannini ${ }^{1,2}$, N. Kassim ${ }^{3}$, and G. Setti ${ }^{1,2}$ \\ 1 Istituto di Radioastronomia - CNR, via P. Gobetti 101, 40129 Bologna, Italy \\ e-mail: iferetti@ira.cnr.it \\ 2 Dipartimento di Astronomia, Univ. Bologna, via Ranzani 1, 40127 Bologna, Italy \\ 3 Naval Research Laboratory, Code 7213, Washington DC 20375, USA
}

Received 23 February 2004 / Accepted 14 April 2004

\begin{abstract}
New radio data at $330 \mathrm{MHz}$ are presented for the rich clusters Abell 665 and Abell 2163, whose radio emission is characterized by the presence of a radio halo. These images allowed us to derive the spectral properties of the two clusters under study. The integrated spectra of these halos between $0.3 \mathrm{GHz}$ and $1.4 \mathrm{GHz}$ are moderately steep: $\alpha_{0.3}^{1.4}=1.04$ and $\alpha_{0.3}^{1.4}=1.18$, for A665 and A2163, respectively. The spectral index maps, produced with an angular resolution of the order of $\sim 1^{\prime}$, show features of the spectral index (flattening and patches), which are indication of a complex shape of the radiating electron spectrum, and are therefore in support of electron reacceleration models. Regions of flatter spectrum are found to be related to the recent merger activity in these clusters. This is the first strong confirmation that the cluster merger supplies energy to the radio halo. In the undisturbed cluster regions, the spectrum steepens with the distance from the cluster center. This is interpreted as the result of the combination of the magnetic field profile with the spatial distribution of the reacceleration efficiency, thus allowing us to set constraints on the radial profile of the cluster magnetic field.
\end{abstract}

Key words. radio continuum: general - galaxies: clusters: general - galaxies: clusters: individual: A665 galaxies: clusters: individual: A2163 - galaxies: intergalactic medium - X-rays: galaxies: clusters

\section{Introduction}

Recent observations of clusters of galaxies have revealed a new and complex scenario in the structure of the intergalactic medium. The clusters are not simple relaxed structures, but are still forming at the present epoch. Substructures, commonly observed in the X-ray distribution of a high number of rich clusters (Henry \& Briel 1993; Burns et al. 1994), are evidence of the hierarchic growth of clusters from the merger of poorer subclusters.

An important problem in cluster phenomenology involves cluster-wide radio halos, whose prototype is Coma C (Giovannini et al. 1993, and references therein). These are extended diffuse radio sources located at the cluster centers, with typical sizes of $\gtrsim 1 h_{50}^{-1} \mathrm{Mpc}$, regular shape, steep radio spectra and no significant polarization. According to recent suggestions, the cluster merger process may play a crucial role in the formation and energetics of these sources (see Giovannini \& Feretti 2002, and references therein).

The origin and evolution of halos is still a matter of debate. Several suggestions for the mechanism transferring energy into the relativistic electron population and for the origin of relativistic electrons themselves have been made: in-situ reacceleration of relativistic electrons by plasma and by shock waves, particle injection from radio galaxies, acceleration out of the thermal pool, secondary electrons resulting from hadronic collisions of relativistic protons with the ICM gas protons, and combinations of these processes (see e.g. Brunetti 2003; Blasi 2003; Petrosian 2003). It is therefore important to carry out new observations aimed at discriminating between these theoretical models.

An important observable in a radio halo is the spectral index distribution. It could allow us to test predictions of these different models, since it reflects the shape of the electron energy distribution. Spectral index maps represent a powerful tool to study the properties of the relativistic electrons and of the magnetic field in which they emit, and to investigate the connection between the electron energy distribution and the ICM. By combining high resolution spectral information and $\mathrm{X}$-ray images it is possible to study the thermal-relativistic plasma connection both on small scales (e.g. spectral index variations vs. clumps in the ICM distribution) and on the large scale (e.g. radial spectral index trends).

The prototypical example of cluster radio halos is the diffuse source Coma C in the Coma cluster (Willson 1970). Nowadays Coma $\mathrm{C}$ is the only radio halo for which a high resolution spectral index image has been presented in the literature (Giovannini et al. 1993). The spectral index trend in Coma $\mathrm{C}$ shows a flat spectrum ${ }^{1}$ in the center $(\alpha \simeq 0.8)$ and a progressive steepening with increasing distance from the center

\footnotetext{
${ }^{1} S_{v} \propto v^{-\alpha}$ through this paper.
} 
Table 1. Observed clusters of galaxies.

\begin{tabular}{lllllllllll}
\hline \hline Name & $z$ & $\begin{array}{l}\text { RA (J2000) } \\
\mathrm{h} \mathrm{m} \mathrm{s}\end{array}$ & $\begin{array}{l}\text { Dec } \\
{ }^{\circ},\end{array}$ & & $\begin{array}{l}T \\
\mathrm{keV}\end{array}$ & $\begin{array}{l}L_{\mathrm{X}}(\mathrm{bol}) \\
\mathrm{erg} \mathrm{s}^{-1}\end{array}$ & $\begin{array}{l}r_{\mathrm{c}} \\
\prime\end{array}$ & $\begin{array}{l}r_{\mathrm{c}} \\
\mathrm{kpc}\end{array}$ & $\begin{array}{l}P_{1.4} \\
\mathrm{~W} \mathrm{~Hz}^{-1}\end{array}$ & $\begin{array}{l}\mathrm{LS} \\
\mathrm{Mpc}\end{array}$ \\
\hline A665 & 0.1818 & 083057.4 & +655114.4 & 9.03 & $4.17 \times 10^{45}$ & 96 & 379 & 0.66 & $6.6 \times 10^{24}$ & 1.8 \\
$\mathrm{~A} 2163$ & 0.203 & 161549.4 & -060900.0 & 13.83 & $1.33 \times 10^{46}$ & 72 & 305 & 0.62 & $3.0 \times 10^{25}$ & 2.9 \\
\hline
\end{tabular}

Notes. Column 1: cluster name; Col. 2: redshift; Cols. 3 and 4: coordinates of the X-ray cluster center from Ebeling et al. (1996); Col. 5: temperature from Allen \& Fabian (1995); Col. 6: bolometric X-ray luminosity from Wu et al. (1999); Cols. 7-9: cluster core radius (angular and linear) and $\beta$ from Birkinshaw et al. (1991) for A665 and Elbaz et al. (1995) form A2163; Cols. 10 and 11: total radio power and maximum linear size at $1.4 \mathrm{GHz}$ from Giovannini \& Feretti (2000) for A665 and Feretti et al. (2001) for A2163.

(up to $\alpha \simeq 1.8$ ). Since the diffusion velocity of relativistic particles is low in relation to their radiative lifetime, the radial spectral steepening cannot be simply due to ageing of radioemitting electrons. Therefore the spectral steepening must be related to the intrinsic evolution of the local electron spectrum and to the radial profile of the cluster magnetic field.

It has been shown (Brunetti et al. 2001) that a relatively general expectation of models invoking reacceleration of relic particles is a radial spectral steepening in the synchrotron emission from radio halos. The steepening, that is difficult to reproduce by other models such as those invoking secondary electron populations, is due to the combined effect of a radial decrease of the cluster magnetic field strength and of the presence of a high energy break in the energy distribution of the reaccelerated electron population. In the framework of reacceleration models the radio spectral index map can be used to derive the physical conditions prevailing in the clusters, i.e. reacceleration efficiency and magnetic field strength.

This method has been successfully applied to the case of Coma C by Brunetti et al. (2001) who applied a two phase reacceleration model and obtained large scale reacceleration efficiencies of the order of $\sim 10^{8} \mathrm{yr}^{-1}$ and magnetic field strengths ranging from $1-3 \mu \mathrm{G}$ in the central regions down to $0.05-$ $0.1 \mu \mathrm{G}$ in the cluster periphery.

In this paper we present the radio images at $90 \mathrm{~cm}$ of Abell 665 and Abell 2163. These clusters host powerful and giant radio halos studied at $20 \mathrm{~cm}$ with the VLA (Giovannini $\&$ Feretti 2000; Feretti et al. 2001). From the comparison between the 20 and $90 \mathrm{~cm}$ data, we derive the spectral index maps of these radio halos and discuss their implications.

We adopt $H_{0}=50 \mathrm{~km} \mathrm{~s}^{-1} \mathrm{Mpc}^{-1}$ and $q_{0}=0.5$. With these values, 1 arcsec corresponds to $3.94 \mathrm{kpc}$ at the distance of A665 and to $4.27 \mathrm{kpc}$ at the distance of A2163.

\section{The clusters}

The general properties of A665 and A2163 are summarized in Table 1. We give below a short description of the radio and $\mathrm{X}$-ray information.

A665. The presence of a radio halo was first reported by Moffet \& Birkinshaw (1989) and confirmed by Jones \& Saunders (1996). The deep VLA image at $20 \mathrm{~cm}$, presented by Giovannini \& Feretti (2000), shows that the radio halo is about 1.8 Mpc in size, with elongation in the SE-NW direction. The radio emission is asymmetric with respect to the cluster center, being brighter and more extended toward NW.
The X-ray emission of this cluster is complex. An acceptable fit of the $\mathrm{X}$-ray brightness profile to a $\beta$ model was obtained by Birkinshaw et al. (1991) using the Einstein IPC data. In the following years, however, Hughes \& Birkinshaw (1994) found that the spatial distribution of the X-ray emitting gas in the ROSAT PSPC data deviates from circular symmetry and argued that a major merger is occurring or had recently occurred in this system. This was confirmed by Gómez et al. (2000), who studied the cluster dynamics on the basis of new redshift measurements and concluded that the velocity distribution is consistent with that predicted in the case of a merger of two similar mass subclusters seen close to the time of core-crossing.

Chandra data presented by Markevitch \& Vikhlinin (2001) indicate that the X-ray contours are elongated in the same direction as the galaxy distribution (Geller \& Beers 1982) and their appearance suggests that the bright core associated with the main galaxy concentration is moving south with respect to the more diffuse cluster component. This is confirmed by their temperature map, which reveals a remarkable shock in front of the cool cluster core, indicating that the core is moving with a relatively high Mach number. The shock is located near the southern boundary of the radio halo. The complex temperature structure is confirmed by the more sensitive data published by Govoni et al. (2004). The low brightness cluster X-ray emission is elongated toward the NW, which is the apparent direction of the merger with a smaller subcluster. This is the same direction as the radio halo emission.

A2163. This cluster is one of the hottest and most X-ray luminous among known clusters. Herbig \& Birkinshaw (1994) first reported the presence of a powerful radio halo, which has been studied in better detail by Feretti et al. (2001). The radio halo, which is one of the most powerful and extended halos known so far, displays a regular shape, slightly elongated in the $\mathrm{E}-\mathrm{W}$ direction. In the $\mathrm{N}-\mathrm{E}$ peripheral cluster region, a diffuse elongated emission, classified as a cluster relic, has been detected. The comparison between the radio emission of the halo and the cluster X-ray emission shows a close structural similarity on the large scale (Feretti et al. 2001).

According to the X-ray morphological study of Elbaz et al. (1995), based on ROSAT data, and the spectroscopic analysis of Markevitch et al. (1996), based on ASCA data, the cluster is likely to be a recent merger. The $\beta$ model gives a good fit to the average X-ray surface brightness profile $\left(\chi^{2}=49\right.$ for 36 d.o.f.), although the cluster is not nicely spherically 
Table 2. Observing log.

\begin{tabular}{llllll}
\hline \hline Name & $\begin{array}{l}v \\
\mathrm{MHz}\end{array}$ & $\begin{array}{l}\Delta v \\
\mathrm{MHz}\end{array}$ & Conf. & Date & $\begin{array}{l}\text { Dur. } \\
\mathrm{h}\end{array}$ \\
\hline $\mathrm{A} 665$ & $321.5 / 327.5$ & 3.125 & $\mathrm{~B}$ & $4 / 2001$ & 3 \\
& “ & “ & $\mathrm{C}$ & $9 / 2001$ & 6 \\
$\mathrm{~A} 2163$ & “ & “ & $\mathrm{D}$ & $12 / 2001$ & 1.5 \\
& “ & “ & $\mathrm{C}$ & $8 / 2001$ & 6 \\
\hline
\end{tabular}

Notes. Column 1: cluster name; Col. 2: observing frequencies; Col. 3 : bandwidth; Col. 4: VLA configuration; Col. 5: month and year of observation; Col. 6: observing time duration.

symmetric and the results obtained depend on the exact region chosen to derive the profile.

Recent Chandra data (Markevitch \& Vikhlinin 2001; Govoni et al. 2004) reveal a complex morphology indicating that the cluster central region is in a state of violent motion. The temperature map is also complex, with variations by at least a factor of 2, suggesting streams of hot and cold gas flowing in different directions, as well as a possible remnant of a cool gas core, surrounded by shock-heated gas.

The structure in the temperature map is too complicated to easily infer the geometry of the merger. The X-ray brightness distribution indicates elongation in the $\mathrm{E}-\mathrm{W}$ direction, suggesting that this could be the merger direction. However, the absence of any sharp features in the X-ray image, and the optical spectroscopic data (Soucail et al., in preparation) point to the possibility that the merger is occurring at a large angle to the sky plane.

A strong Sunyaev-Zel'dovich (SZ) effect has been reported in this cluster (Holzapfel et al. 1997; Désert et al. 1998).

\section{Radio observations}

Radio observations were obtained with the VLA at 321.5 and $327.5 \mathrm{MHz}$ in several different configurations (see Table 2).

Observations were performed in spectral line mode, with 32 channels, to reduce the effects of bandwidth smearing and allow for more accurate excision of narrow-band external RFI. For both targets, the bandpass calibration was performed from observations of Cygnus A, whereas 3C 286 was used for initial amplitude and phase calibration and to set the VLA flux density scale. The sources $0834+555$ and $1605-175$ were observed as secondary phase calibrators for A665 and A2163, respectively. The entire field was imaged using the AIPS program IMAGR in 3D mode and selfcalibrated using CALIB. This procedure was performed using the task VLALB, kindly provided by W. Cotton. Further data editing, loops of selfcalibration and widefield image deconvolution were used to mitigate confusion and achieve the maximum sensitivity.

For A2163, the data from the B and C configurations have been first reduced and self-calibrated separately, then combined to produce the final image. For A665, the radio halo is not detected in the B array observation, whereas the data from the $\mathrm{D}$ configuration are too noisy to be useful. Therefore the final image for this cluster is essentially obtained with the $\mathrm{C}$ configuration data.

The achieved sensitivities are $0.9 \mathrm{mJy} /$ beam for A665 and $0.4 \mathrm{mJy} / \mathrm{beam}$ for A2163. These are somewhat higher than the theoretically expected thermal noise levels $(\sim 0.55 \mathrm{mJy} / \mathrm{beam}$ in 1 hour integration), probably because of confusion and of broad-band radio frequency interference, mainly VLA-generated.

\section{Results}

The final images of the radio halos in A665 and A2163 at $90 \mathrm{~cm}$ are presented in Figs. 1 and 2, respectively.

In A665, the radio emission is rather centrally condensed, with low brightness features extending to the south and to the north. The extreme narrow region of the southern extension is due to the blend of discrete sources, as deduced by the comparison with the $20 \mathrm{~cm}$ image. Therefore the diffuse halo emission is asymmetric toward the $\mathrm{N}-\mathrm{NW}$ direction. The $\mathrm{X}-$ ray gas distribution is asymmetric in the same direction (see e.g. the Chandra image by Markevitch \& Vikhlinin 2001). The total flux of the halo, excluding the unrelated sources, is $197 \pm 6 \mathrm{mJy}$. This value leads to a total spectral index $\alpha_{0.3}^{1.4}=1.04 \pm 0.02$. The central strongest halo region has a quite regular shape, with a $F W H M$ of about $180^{\prime \prime}$, corresponding to $\sim 700 \mathrm{kpc}$. We note that this is the cluster region approximately within 1 core radius (see Table 1 ).

In A2163, the structure of the diffuse emission is remarkably similar to that detected at $1.4 \mathrm{GHz}$, with a similar extent. The total flux, after subtraction of discrete sources is $861 \pm$ $10 \mathrm{mJy}$. This leads to a total spectral index $\alpha_{0.3}^{1.4}=1.18 \pm 0.04$. Feretti et al. (2001) estimated a spectral index of $\sim 1.6 \pm 0.3$ by comparing the total flux densities at the two nearby frequencies of $1.365 \mathrm{MHz}$ and $1.465 \mathrm{MHz}$ within the $20 \mathrm{~cm}$ radio band. Even allowing for the large errors, this could be indication of a spectral steepening above $\sim 1.4 \mathrm{GHz}$.

The extended feature that was classified as a relic by Feretti et al. (2001) is indicated in Fig. 2 by the letter " $R$ ". It has a total flux of $82 \pm 2 \mathrm{mJy}$, and therefore a total spectral index $\alpha_{0.3}^{1.4}=1.02 \pm 0.04$.

Feretti et al. (2001) also discussed the possibility that this feature may be part of a wide-angle-tailed (WAT) radio source with the nucleus coincident with the strong radio source $\mathrm{J} 1616-061$, at position $\mathrm{RA}=16^{\mathrm{h}} 16^{\mathrm{m}} 22.2^{\mathrm{s}}$, Dec $=-06^{\circ} 06^{\prime} 34^{\prime \prime}$ (i.e. to the S-E of the relic). Arguments against this interpretation were that the WAT radio source would have the exceptionally large size of about $1.3 \mathrm{Mpc}$ (assuming it is a cluster radio galaxy) and that WAT sources are usually located at the cluster centers. The present observations lend support to the interpretation that this feature is a relic. In fact, the strong radio source J1616-061 seems disconnected from the source " $R$ ". More important, we derive that the spectrum of source " $R$ " is flatter at its peak location, and shows slight steepening toward the edges (see Fig. 4), whereas a connection to the source J1616-061 would imply a progressive spectral steepening from its nucleus to the end of the tail. From the morphological and spectral trend, the source $\mathrm{J} 1616-061$ is likely to be a tailed radio galaxy, extended toward the south. 


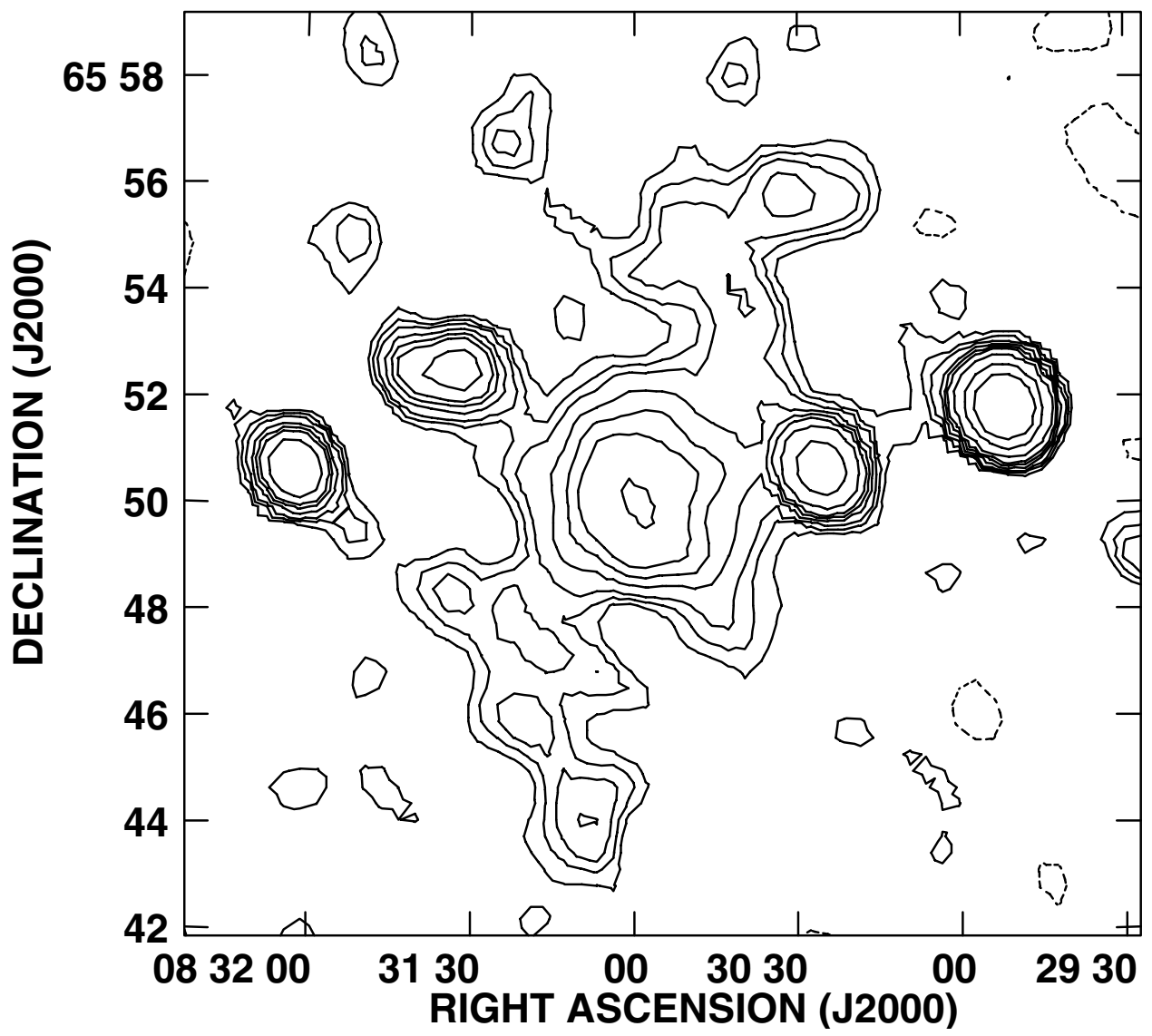

Fig. 1. Radio map at $90 \mathrm{~cm}$ of $\mathrm{A} 665$. The beam $F W H M$ is $68^{\prime \prime} \times 59^{\prime \prime}$ in PA $=25^{\circ}$. Contour levels are $-2,2,3,4,6,8,10,15,30,50 \mathrm{mJy} /$ beam.

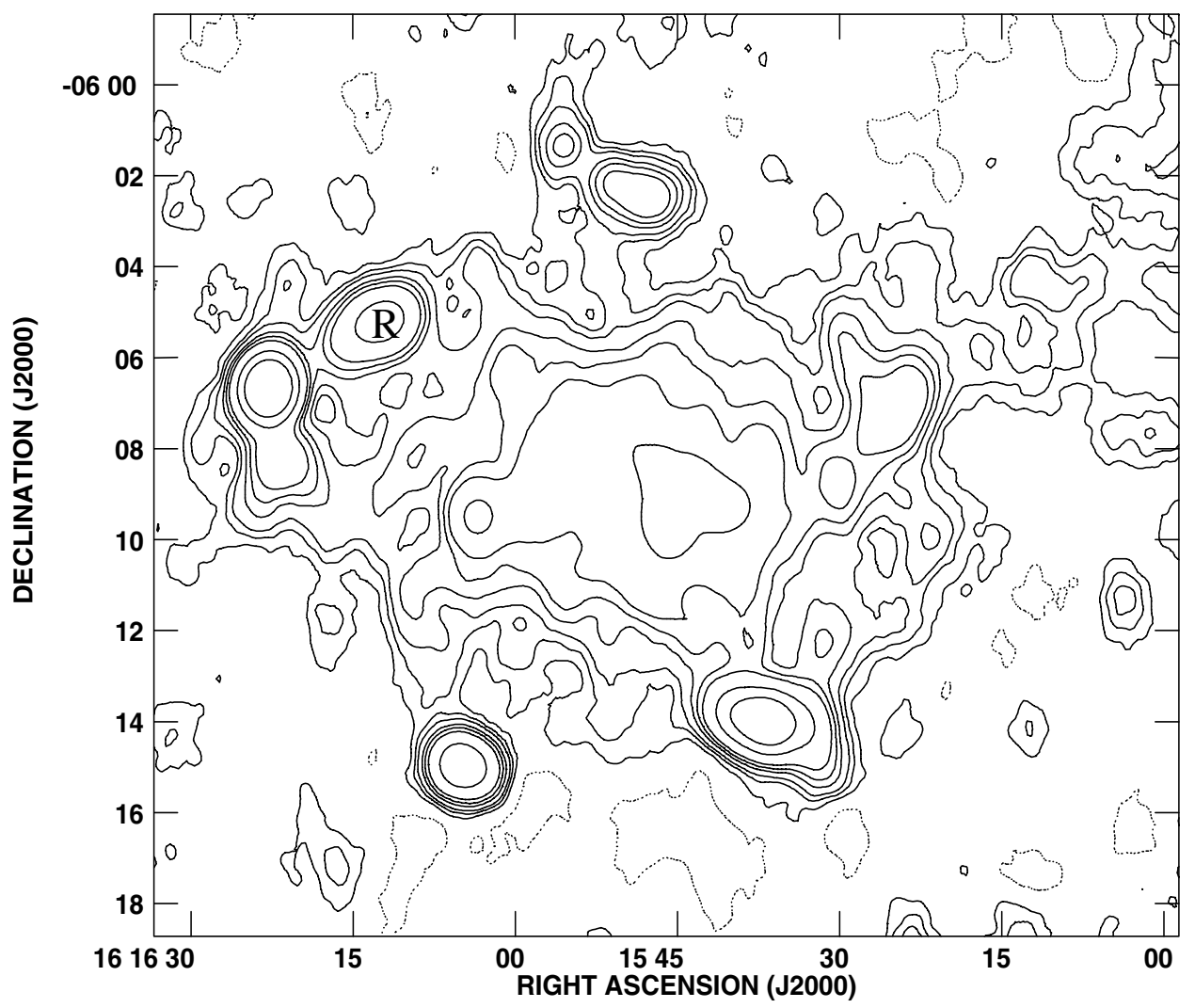

Fig. 2. Radio map at $90 \mathrm{~cm}$ of $A 2163$. The beam $F W H M$ is $57^{\prime \prime} \times 51^{\prime \prime}$ in $\mathrm{PA}=2^{\circ}$. Contour levels are $-1,1,2,3,5,7,10,25,50 \mathrm{mJy} / \mathrm{beam}$. The letter " $R$ " indicates the radio relic (see text). 


\section{Spectral index maps}

The spectral index maps of A665 and A2163 were obtained by comparing the $90 \mathrm{~cm}$ and $20 \mathrm{~cm}$ images, produced with the same beam and cellsize. The data at $90 \mathrm{~cm}$ provide a better coverage of short spacings than those at $20 \mathrm{~cm}$. Indeed the minimum spacing at $90 \mathrm{~cm}$ is $\sim 35 \lambda$, whereas at $20 \mathrm{~cm}$ it is $\sim 150 \lambda$. However, we are confident that the spectral comparison is not affected for the following reasons: i) the total structures of the halos in A665 and A2163 are extended about 10' and about $11.5^{\prime}$, respectively. Therefore, the structure of both halos is expected to be fully imaged from the $20 \mathrm{~cm}$ observations, which are blind to structures larger than $\sim 15^{\prime}$; ii) the shortest baselines at $90 \mathrm{~cm}$ are provided by a few interferometers only ( $\mathrm{C}$ array), so the coverage of short spacings is poor at $90 \mathrm{~cm}$; iii) the total spectral index between 90 and $20 \mathrm{~cm}$ is not very steep $(\alpha \sim 1)$. If some flux was missed in the $20 \mathrm{~cm}$ data the real spectrum should be flatter, in contrast to values of spectra of radio halos; iv) the $20 \mathrm{~cm}$ map is much more sensitive than that at $90 \mathrm{~cm}$.

A665. The spectral index map is clumpy (top panel of Fig. 3). The spectrum in the central halo region is rather constant, with spectral index values between 0.8 and 1.2 within one core radius from the cluster center (i.e. within $\sim 95^{\prime \prime}$ ). In the northern region of lower radio brightness, the spectrum is flatter than in the southern halo region.

Starting from the approximate radio peak position, we obtained profiles of the spectral index trend by averaging the values of the spectral index within small sectors around the two directions marked by dashed lines. The spectrum in the N-W direction flattens up to a distance of about $200^{\prime \prime}$ from the center (Fig. 3, bottom left panel, green triangles). This is the region where asymmetric extended X-ray emission is present, indicating the existence of an ongoing merger with another cluster. The gas temperature in this region (Markevitch \& Vikhlinin 2001; Govoni et al. 2004) shows strong variations, from about $12 \mathrm{keV}$ in the $\mathrm{N}-\mathrm{E}$ to about $8 \mathrm{keV}$ in the $\mathrm{S}-\mathrm{W}$. The spectral index flattening follows the X-ray morphology, but there is no one-to-one correspondence with the value of the gas temperature. Therefore, it seems that this region, which is presently strongly influenced by the merger, is a shocked region, where the gas at different temperatures is still in the process of mixing.

In defining a profile in the southern cluster region, we tried to avoid the region where there is a possible contamination of discrete sources. The spectrum in this direction (Fig. 3, bottom left panel, red dots) steepens significantly from the center to the periphery. The spectral index increase from $\alpha \sim 1$ to $\alpha \gtrsim 2$ is gradual and rather regular, and occurs on a scale of less than 3 cluster core radii. The region of constant spectral index at distance between $120^{\prime \prime}$ and $220^{\prime \prime}$ from the center is located NE of the discrete source and could be affected by the presence of this source. We note that this path crosses the region of the hot shock detected by Chandra at the southernmost edge of the radio halo (Markevitch \& Vikhlinin 2001). The shock is located at about $100^{\prime \prime}$ from the center along the profile. No significant spectral flattening is detected at this position.
A2163. The spectral index map, shown in the top panel of Fig. 4, is clumpy in the central cluster region, but it is rather constant within one core radius (corresponding to $\sim 70^{\prime \prime}$ ), showing spectral index values between 1 and 1.1. On a larger scale, there is evidence that the western halo region is flatter than the eastern one. Particularly, there is a vertical region crossing the cluster center and showing flatter spectrum, with a clear evidence of spectral flattening both at the northern and at the southern halo boundaries.

Radial profiles of the spectral index along two interesting directions (see dashed lines) have been obtained, as in A665, by averaging the values of the spectral index within small sectors around the two directions. The spectral index profile along the N-S direction (Fig. 4, bottom left panel, green triangles) is globally rather flat and shows a significant flattening at about $300^{\prime \prime}$ from the center (note that the strong flattening of the two last points is due to the presence of a discrete radio source). In the eastern cluster region, in the area free of discrete sources, the spectrum becomes progressively steeper from the center to the periphery. This is well seen in the profile along the S-E direction, given by red dots in the left bottom panel of Fig. 4. The spectral steepening is weaker than in A665, and occurs over a much larger scale. The north-south region of flatter spectrum coincides with the region of highest temperature detected from Chandra. Although the geometry of the merger cannot be established by the X-ray Chandra data (Markevitch \& Vikhlinin 2001; Govoni et al. 2004), the region of flatter spectrum is likely related to the strong dynamical activity at the cluster center. The N-S extent of the region with flat spectrum is in support of a merger occurring in the E-W direction, as indicated by the X-ray brightness distribution. The complexity of the merger is, however, reflected in the complexity of the spectral index map.

\section{Discussion}

\subsection{Spectral index behaviour}

The maps reported in Figs. 3 and 4 indicate the existence of patches of different spectral index values, with significant variations on scales of the order of the observing beam ( $200 \mathrm{kpc})$. This suggests a complex shape of the electron spectrum, as generally expected in the case of particle reacceleration.

The regions currently influenced by an ongoing merger show a different behaviour with respect to the relatively undisturbed regions. Regions of flatter spectra are indication of the presence of more energetic radiating particles, and/or of a larger value of the local magnetic field strength. As qualitatively expected by electron reacceleration models, relatively flatter regions of synchrotron spectral index are found in regions currently influenced by merger processes, while a general radial spectral steepening is found in presently undisturbed cluster regions.

Numerical simulations show that the intensity of the cluster magnetic field can be significantly enhanced during ongoing mergers (Roettiger et al. 1999). In addition mergers are believed to be responsible for the injection of cluster turbulence (Norman \& Bryan 1999) which is most likely connected to 

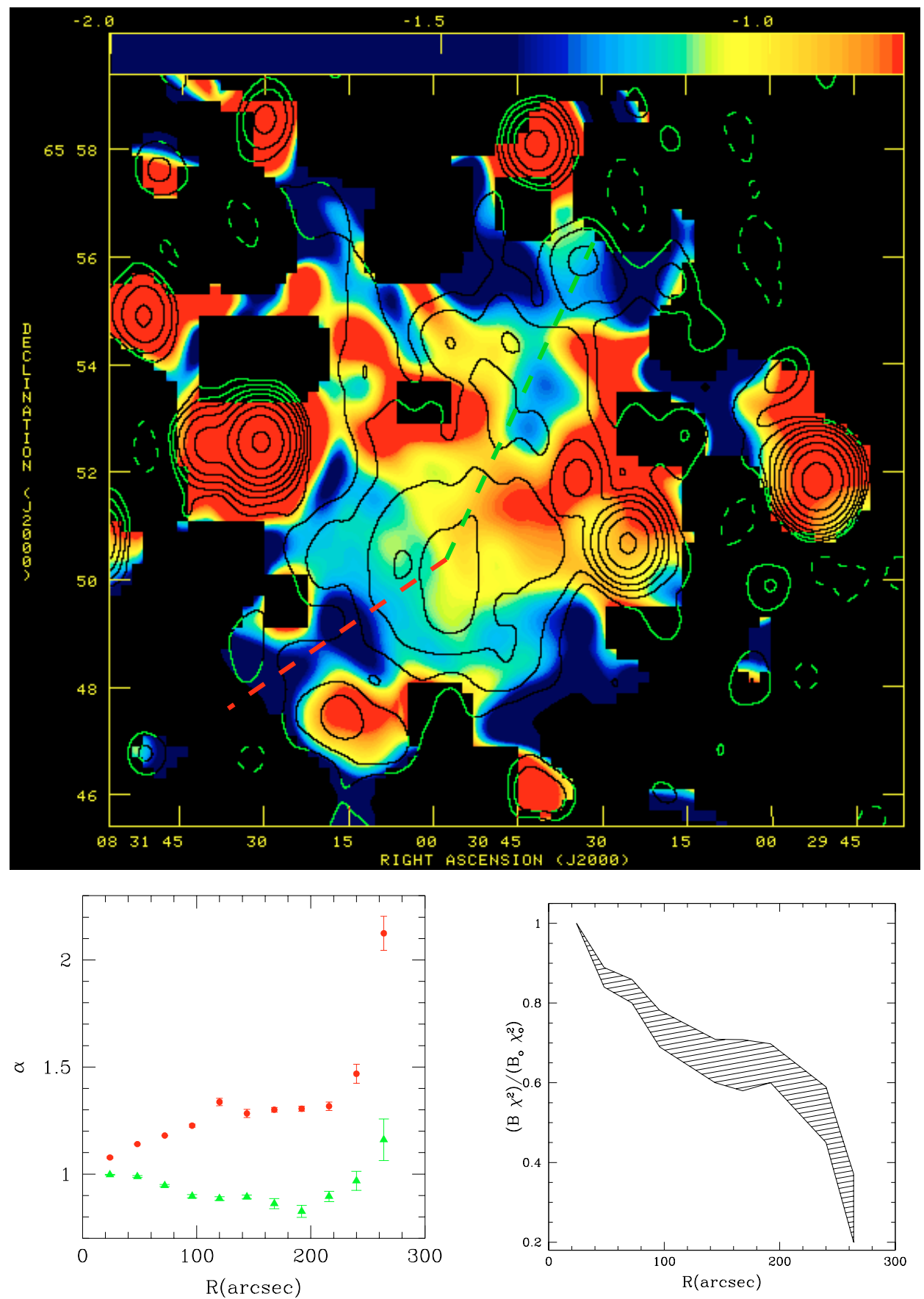

Fig. 3. Top panel: color-scale image of the spectral index between $0.3 \mathrm{GHz}$ and $1.4 \mathrm{GHz}$ of A665, obtained with a resolution of $68^{\prime \prime} \times 59^{\prime \prime}$ $\left(\mathrm{PA}=25^{\circ}\right) F W H M$. The contours indicate the radio emission at $20 \mathrm{~cm}$ (from Giovannini \& Feretti 2000) at levels of $-0.2,0.2,0.4,0.8,1.5,3.0$, $6.0,12.0,25.0 \mathrm{mJy} /$ beam (beam $=52^{\prime \prime} \times 42^{\prime \prime}$ ). Bottom left panel: radial profiles of the spectral index along the two directions indicated by the dashed lines in the spectral index map: the green triangles report the profile toward the $\mathrm{N}-\mathrm{W}$ (green dashed line), the red dots give the profile toward the $\mathrm{S}-\mathrm{E}$ (red dashed line). In both profiles, the origin of the distance scale is the approximate radio peak position at the cluster center $\left(\mathrm{RA}_{2000}=08^{\mathrm{h}} 30^{\mathrm{m}} 56.0^{\mathrm{s}}, \mathrm{Dec}_{2000}=65^{\circ} 50^{\prime} 09.7^{\prime \prime}\right)$. Bottom right panel: radial profile of the product between the magnetic field and the electron reacceleration coefficient, normalized to its value at the custer center.

the acceleration of relativistic electrons (Brunetti et al. 2001; Fujita et al. 2003). Thus, in general, in the regions directly influenced by ongoing mergers the synchrotron spectrum may become flatter due to an increase of both the magnetic field strength and the acceleration efficiency.

Indeed the regions with flatter spectra are observed along profiles which appear to trace the geometry of recent merger activity as suggested by X-ray maps. Our results prove that the radio spectral index can be a powerful tracer of the current physical properties of clusters, and confirms the importance of cluster merger in the energetics of relativistic particles responsible for the halo radio emission. On the other hand, the spectral index steepens progressively with the distance from the cluster center in the region not presently affected by the merging 

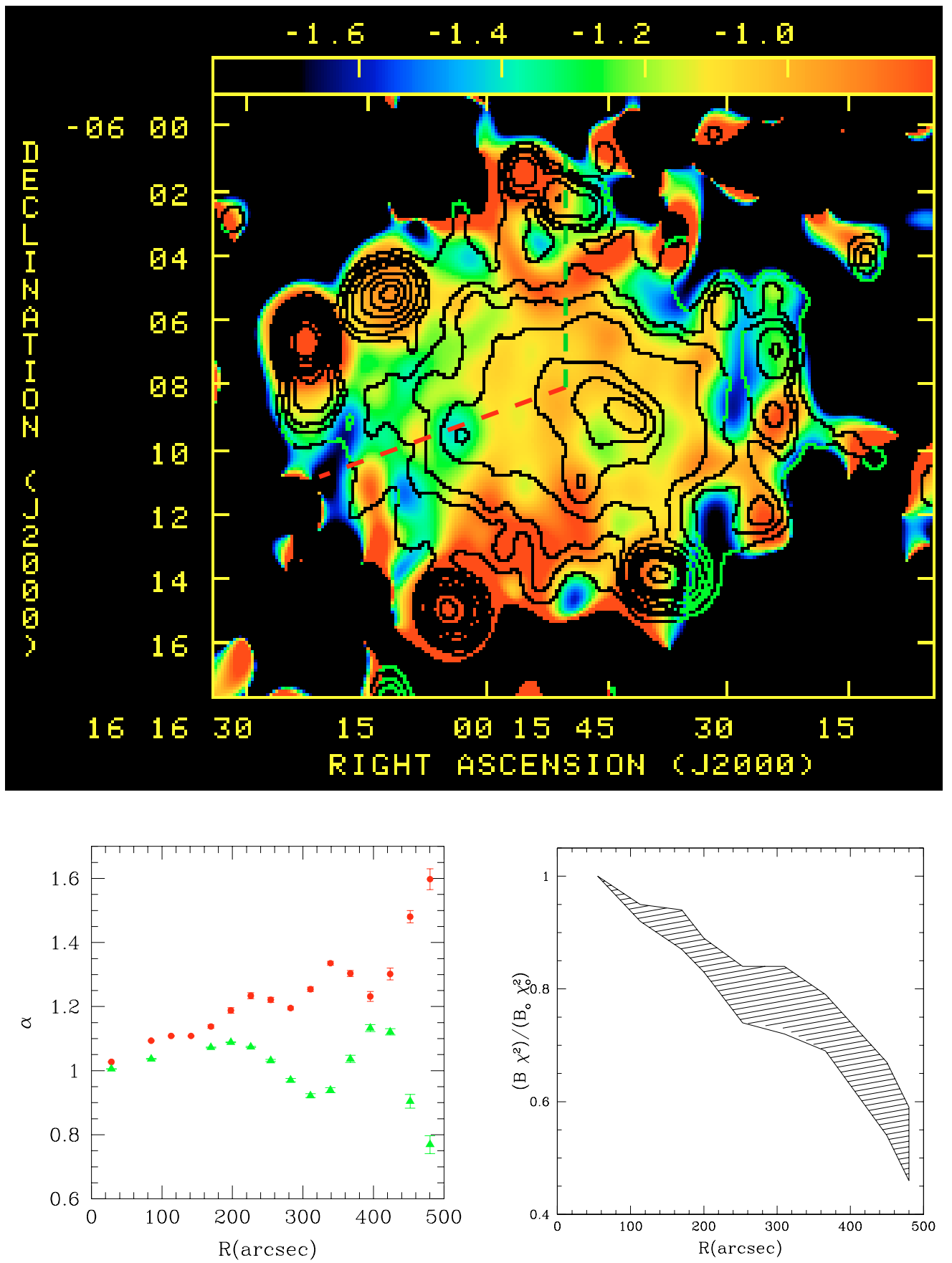

Fig. 4. Top panel: color-scale image of the spectral index between $0.3 \mathrm{GHz}$ and $1.4 \mathrm{GHz}$ of A2163, obtained with a resolution of $60^{\prime \prime} \times 51^{\prime \prime}$ $\left(\mathrm{PA}=0^{\circ}\right) F W H M$. The contours indicate the radio emission at $20 \mathrm{~cm}$ (from Feretti et al. 2001) at levels of 0.2, 0.5, 0.8, 1.5, 3.0, 5.0, 7.0, $9.0,15.0,25.0 \mathrm{mJy} /$ beam (beam $=60^{\prime \prime} \times 45^{\prime \prime}$ ). Bottom left panel: radial profiles of the spectral index along the two directions indicated by the dashed lines in the spectral index map: the green triangles report the profile toward the $\mathrm{N}$ (green dashed line), the red dots give the profile toward the S-E (red dashed line). In both profiles, the origin of the distance scale is the approximate center of the radio emission $\left(\mathrm{RA}_{2000}=16^{\mathrm{h}} 15^{\mathrm{m}} 51.2^{\mathrm{s}},-06^{\circ} 08^{\prime} 48.1^{\prime \prime}\right.$, note that the radio peak to the east is contaminated by discrete sources). Bottom right panel: radial profile of the product between the magnetic field and the electron reacceleration coefficient, normalized to its value at the cluster center.

processes. This is another indication that the energy of relativistic particles is sensitive to the effect of mergers.

It is worth noticing that there is no evidence of spectral flattening at the location of the hot shock detected in A665 (Markevitch \& Vikhlinin 2001). This is consistent with the fact that shocks in major mergers are too weak for particle acceleration (Gabici \& Blasi 2003) and indeed the Mach number of the shock in A665 is 2 (Markevitch \& Vikhlinin 2001). Our result supports the scenario that cluster turbulence might be the major responsible for the supply of energy to the radiating electrons. 
We have computed the physical conditions within the radio halos in $\mathrm{A} 665$ and $\mathrm{A} 2163$, using standard formulae (Pacholczyk 1970) with the standard assumptions of equal energy density in protons and electrons, a magnetic field filling factor of 1 , a spectrum extending from $10 \mathrm{MHz}$ to $100 \mathrm{GHz}$ with the integrated spectral index obtained in Sect. 4. The minimum energy density in A665 is $u_{\text {min }}=2.8 \times 10^{-14} \mathrm{erg} \mathrm{cm}^{-3}$, and the corresponding equipartition magnetic field is $B_{\mathrm{eq}}=$ $5.5 \times 10^{-7} \mathrm{G}$. In A2163, we obtain $u_{\min }=3.8 \times 10^{-14} \mathrm{erg} \mathrm{cm}^{-3}$, and an equipartition magnetic field $B_{\text {eq }}=6.4 \times 10^{-7} \mathrm{G}$.

We have attempted the evaluation of the energy supplied to the halos in the regions of flatter spectral index, by matching the reacceleration gains and the radiative losses of the radio emitting electrons. In regions of identical volume and same brightness at $0.3 \mathrm{GHz}$, a flattening of the spectral index from 1.3 to 0.8 implies that the energy injected into the electron population is larger by a factor of $\sim 2.5$.

Alternatively, if electrons have been reaccelerated in the past, and they are simply ageing, we can consider that the flatter spectrum reflects a spectral cutoff at higher energies. Particles with a frequency cutoff at $v_{\mathrm{b}}=1.4 \mathrm{GHz}$ have a lifetime of about $4.5 \times 10^{7} \mathrm{yr}$, whereas for $v_{\mathrm{b}}=0.3 \mathrm{GHz}$ the particle lifetime is $9.4 \times 10^{7} \mathrm{yr}$, assuming a magnetic field of about $0.5 \mu \mathrm{G}$. Therefore it follows that the electrons in the flat spectrum regions have been reaccelerated more recently.

\subsection{Implications on particle acceleration and cluster magnetic field}

In the primary reacceleration scenario the electrons are accelerated up to a maximum energy which is given by the balance between acceleration efficiency and energy losses. As a consequence a break (or cut-off) is expected in the synchrotron spectrum emitted by these electrons. The presence of spectral steepenings and flattenings implies that the frequency of such a synchrotron break is relatively close to the range $0.3-1.4 \mathrm{GHz}$ for a relevant fraction of the cluster volume.

If Fermi-like acceleration processes are efficient in the cluster volume, the synchrotron break frequency $v_{\mathrm{b}}$ is related to the magnetic field $B$ as:

$v_{\mathrm{b}}(r) \propto \chi^{2}(r) \begin{cases}B(r), & \text { if } B(r) \ll B_{\mathrm{IC}} \\ B^{-3}(r), & \text { if } B(r) \gg B_{\mathrm{IC}}\end{cases}$

Where $\chi(r)=\tau_{\text {acc }}^{-1}$ is the acceleration rate $(\mathrm{d} p / \mathrm{d} t=\chi p)$, and $B_{\mathrm{IC}}$ is the IC equivalent magnetic field $\sim 3 \mu \mathrm{G}$.

The equipartition magnetic field evaluated in the previous section is $\ll B_{\text {IC }}$ in the present clusters, thus we expect $v_{\mathrm{b}}(r) \propto \chi^{2}(r) B(r)$. We remark that this value is averaged on the radio halo volume, therefore it represents the large scale field. Allowing for a decrease of $B$ with $r$, it follows that a roughly constant acceleration efficiency results in a systematic steepening of the synchrotron spectrum with $r$, simply because at a given frequency higher energy electrons are needed to emit in the lower field intensity. This steepening effect would be further enhanced if the reacceleration efficiency increases toward the central regions.
In the bottom right panels of Figs. 3 and 4, we report the trends of $B \chi^{2}$ for A665 and A2163, respectively, as a function of $r$, for the undisturbed regions. Each profile, normalized to its value at the cluster center, reflects the trend of the spectral index (shown by red dots in the bottom left panels of the same figures). To obtain the radial dependence of the break frequency (and thus $B \chi^{2}$ ) we have fitted the radial behaviour of the synchrotron spectral index with synchrotron spectra emitted by a population of accelerated electrons. In the calculations of the electron spectra we have assumed that the electrons have been accelerated at the same time. To constrain the magnetic field intensity, we have used a range of electron spectra obtained by assuming different strengths of the acceleration coefficient and different duration times of the acceleration phase. We have also included the uncertainties due to the deprojection of the synchrotron spectra with distance.

In each cluster, the trend of $B \chi^{2}$ represents the profile of the magnetic field strength, in the case that the reacceleration is constant throughout the cluster. Under this hypothesis, we can compare the profile of the magnetic field with that obtained in the case of a frozen-in magnetic field, $B \propto n_{\text {th }}^{2 / 3}$, where $n_{\text {th }}$ is the thermal plasma density. This profile is expected if the intensity of the magnetic field results from the compression of the thermal plasma during the cluster gravitational collapse. We derive that this trend is not a good representation of the observations, expecially in the case of A2163. Indeed, the intensity of the frozen-in magnetic field decreases by $50 \%$ at 130 " for A665, and at $110^{\prime \prime}$ for A2163, much steeper than estimated by the spectral behaviour. We note, however, that in A665 and A2163 the $\beta$ model approximation for the distribution of $n_{\text {th }}$ may be a too rough representation of the gas distribution, owing to the presence of gas perturbations as a result of strong dynamical evolution. On the other hand, detailed MHD numerical simulations show that the radial behaviour of the magnetic field with radius may diverge from the prediction of a frozen-in B model, resulting flatter in the central regions, and steeper in the external regions (Dolag et al. 1999, 2002).

Radial profiles of the cluster magnetic fields have been derived in the literature for Coma by modeling the spectral steepening (Brunetti et al. 2001) and for A119, using the Rotation Measure of cluster radio galaxies (Dolag et al. 2001). In both clusters, the magnetic field profiles decline with the distance and are not too different from those expected by the frozen-in B model.

We finally remark that the hypothesis of constant reacceleration efficiency in the cluster volume may not be valid, according to the following arguments; i) first results from numerical simulations (Norman \& Bryan 1999) indicate that the injection of turbulence in clusters is not homogeneous and occurs on very different scales in different regions; ii) detailed calculations of particle acceleration due to Alfvèn waves show that, under reasonable assumptions, the acceleration efficiency slightly increases with distance from the cluster center (Brunetti et al. 2003); iii) the radiative losses of electrons in the innermost cluster regions may be strongly increased if the magnetic field is larger than the equipartition value, in particular if it is of the order of $B_{\text {IC }}$ (e.g. Kuo et al. 2003). 
In general, the ongoing violent mergers in A665 and A2163 are likely to play a crucial role in determining the conditions of the radiating particles and the magnetic field in these clusters.

\section{Conclusions}

In this paper we have presented new images at $0.3 \mathrm{GHz}$ of the radio halos in the clusters A665 and A2163 and we have derived the spectral index maps between 0.3 and $1.4 \mathrm{GHz}$, using maps already published at $1.4 \mathrm{GHz}$.

Our results show that the integrated radio spectra of these halos are moderately steep in this frequency range: $\alpha_{0.3}^{1.4}=1.04$ and $\alpha_{0.3}^{1.4}=1.18$, for A665 and A2163, respectively.

The spectral index maps, produced with an angular resolution of the order of $\sim 1^{\prime}$, show a clumpy distribution with significant variations, which are indication of a complex shape of the radiating electron spectrum, and are therefore in support of halo models invoking the reacceleration of relativistic particles.

We find that the regions of flatter spectrum appear to trace the geometry of recent merger activity as suggested by X-ray maps. These results prove that the radio spectral index can be a powerful tracer of both the current physical properties and past history of clusters.

We find no evidence of spectral flattening at the location of the hot shock detected in A665 (Markevitch \& Vikhlinin 2001). This favours the scenario that cluster turbulence might be the major responsible for the electron reacceleration.

In the undisturbed cluster regions, the spectrum steepens with the distance from the cluster center. Brunetti et al. (2001) explain the spectral steepening in the framework of electron reacceleration models, as due to the combined effect of a radial decrease of the cluster magnetic field strength and of the presence of a high energy break in the energy distribution of the reaccelerated electron population. This is more difficult to reproduce by other models, such as those requiring secondary electron populations.

The spectral steepening detected in A665 and A2163 in the direction not presently affected by the merging processes allowed us to constrain the profile of the product between the cluster magnetic field and the reacceleration efficiency, $B \chi^{2}$, under simple assumptions.

The magnetic field profile in both clusters is flatter than predicted in the case of a constant reacceleration and magnetic field frozen to the cluster thermal gas.

The ongoing violent mergers may play a crucial role in determining the conditions of the radiating particles and of the magnetic fields in clusters.

Acknowledgements. The National Radio Astronomy Observatory is operated by Associated Universities, Inc., under contract with the National Science Foundation. Basic research in radio astronomy at the Naval Research Laboratory is supported by the Office of Naval Research. This work has been partially funded by the Italian Space Agency (ASI).

\section{References}

Allen, S. W., Fabian, A. C., Edge, A. C., Böhringer, H., \& White, D. A. 1995, MNRAS, 275, 741

Birkinshaw, M., Hughes, J. P., \& Arnaud, K. A. 1991, ApJ, 379, 466

Blasi, P. 2003, in Matter and energy in clusters of galaxies, ed. S. Bowyer, \& C.-Y. Hwang, ASP Conf. Ser., 203

Brunetti, G., Setti, G., Feretti, L., \& Giovannini, G. 2001, MNRAS, 320,365

Brunetti, G. 2003, in Matter and energy in clusters of galaxies, ed. S. Bowyer, \& C.-Y. Hwang, ASP Conf. Ser., 349

Brunetti, G., Blasi, P., Cassano, R., \& Gabici, S. 2003, MNRAS, in press [arXiv: astro-ph/0312482]

Burns, J. O., Rhee, G., Owen, F. N., \& Pinkey, J. 1994, ApJ, 423, 94

Désert, F.-X., Benoit, A., Gaertner, S., et al. 1998, New Astron., 655, 669

Dolag, K., Bartelmann, M., \& Lesch, H. 1999, A\&A, 348, 351

Dolag, K., Schindler, S., Govoni, F., \& Feretti, L. 2001, A\&A, 378, 777

Dolag, K., Bartelmann, M., \& Lesch, H. 2002, A\&A, 387, 383

Ebeling, H., Voges, W., Böhringer, H., et al. 1996, MNRAS, 281, 799

Elbaz, D., Arnaud, M., \& Böhringer, H. 1995, A\&A, 293, 337

Feretti, L., Fusco-Femiano, R., Giovannini, G., \& Govoni, F. 2001, A\&A, 373, 106

Fujita, Y., Takizawa, M. C. L., \& Sarazin, C. L. 2003, ApJ, 584, 190

Gabici, S., \& Blasi, P. 2003, ApJ, 583, 695

Geller, M. J., \& Beers, T. C. 1982, PASP, 94, 421

Giovannini, G., \& Feretti, L. 2000, New Astron., 5, 335

Giovannini, G., \& Feretti, L. 2002, in Merging processes of galaxy clusters, ed. L. Feretti, I. M. Gioia, \& G. Giovannini, ASSL (Kluwer Ac. Publish.), 197

Giovannini, G., Feretti, L., Venturi, T., Kim, K.-T., \& Kronberg, P. P. 1993, ApJ, 406, 399

Gómez, P. L., Hughes, J. P., \& Birkinshaw, M. 2000, ApJ, 540, 726

Govoni, F., Markevitch, M., Vikhlinin, A., et al. 2004, ApJ, 605, 695

Henry, J. P., \& Briel, U. G. 1993, Adv. Space Res., 13, 191

Herbig, T., \& Birkinshaw, M. 1994, BAAS, 26, 1403

Holzapfel, W. L., Ade, P. A. R., Church, S. E., et al. 1997, ApJ, 481, 35

Hughes, J. P., \& Birkinshaw, M. 1994, in The soft X-ray cosmos, AIP Conf. Proc., 313, ed. E. M. Schlegel, \& R. Petre (New York: AIP), 378

Jones, M., \& Saunders, R. 1996, in Röntgenstrahlung from the Universe, ed. H. U. Zimmermann, J. E. Trümper, \& H. Yorke, MPE Report, 263, 553

Kuo, Ping-Hung, Hwang, Chorng-Yuan, \& Ip, Wing-Huen 2003, ApJ, 594, 732

Markevitch, M., \& Vikhlinin, A. 2001, ApJ, 563, 95

Markevitch, M., Mushotzky, R., Inoue, H., et al. 1996, ApJ, 456, 437

Moffet, A. T., \& Birkinshaw, M. 1989, AJ, 98, 1148

Norman, M. L., \& Bryan, G. L. 1999, in The radio galaxy Messier 87, ed. H. J. Röser, \& K. Meisenheimer, Lect. Notes Phys., 530, 106

Pacholczyk, A. G. 1970, Radio Astrophysics (San Francisco: Freeman and Co.)

Petrosian, V. 2003, in Matter and Energy in Clusters of Galaxies, ed. S. Bowyer, \& C.-Y. Hwang, ASP Conf. Ser., 203

Roettiger, K., Stone, J. M., \& Burns, J. O. 1999, ApJ, 518, 594

Willson, M. A. G. 1970, MNRAS, 151, 1

Wu, X-., Xue, Y.-J., \& Fang, L.-Z. 1999, ApJ, 524, 22 